\title{
Ultrasound array measurement system for multi-plane and multi-component flow mapping in liquid metals
}

\author{
R. Nauber ${ }^{1}$, L. Büttner ${ }^{1}$, M. Burger ${ }^{1}$, M. Neumann ${ }^{1}$, S. Eckert ${ }^{2}$, O. Pätzold ${ }^{3}$, \\ J. W. Czarske ${ }^{1}$ \\ ${ }^{1}$ Laboratory of Measurement and Testing Techniques, Faculty of Electrical and Computer \\ Engineering, Technische Universität Dresden, 01062 Dresden, Germany \\ ${ }^{2}$ Helmholtz-Zentrum-Dresden-Rossendorf, 01314 Dresden, Germany \\ ${ }^{3}$ Institut für Nichteisen-Metallurgie und Reinststoffe, TU Bergakademie Freiberg, \\ 09599 Freiberg, Germany
}

\begin{abstract}
:
Flows of metal melts are of outstanding importance for a variety of technological processes, especially for steel production, such as the continuous casting process. In recent developments, the application of magnetic fields is investigated to influence the flow in a defined way and to optimize the quality of the resulting product. Experimental flow investigations are indispensable to understand the interaction between the magnetic fields and the induced flows. The ultrasound pulse Doppler technique is appropriate for flow investigations. However, the available devices employ only a strongly limited number of transducers, which often is not sufficient for a flow mapping. In order to overcome these limitations a dual-plane, dual-component ultrasound array Doppler velocimeter for mapping of transient flow phenomena is presented. It utilizes four sensor arrays consisting of 25 single element transducers along a line of $67 \mathrm{~mm}$. The system combines a spatial resolution of up to $3 \mathrm{~mm}$ with a temporal resolution of $30 \mathrm{~Hz}$ using electronic beam traversing and time division multiplex. The modular realization of the measurement system allows flexible sensor configuration, e. g. four planes can be measured with one velocity component, two planes with two components or two lines with three components. Those capabilities are demonstrated by measurements in a magnetically stirred metal melt at room temperature.
\end{abstract}

Key words: Ultrasound Doppler array velocimetry, liquid metals, magnetohydrodynamics, rotating magnetic fields

\section{Introduction}

Influencing the flow of metal melts is very important for a variety of industrial processes, e.g. continuous steel casting and semiconductor crystal pulling, in order to improve the quality of the resulting product. The application of steady and/or time-varying magnetic fields allows achieving this without mechanical contact to the melt.

In order to understand these interactions between magnetic fields and fluid flows, experimental flow investigations are performed in field of magnetohydrodynamics (MHD). It is common practice to conduct scaled model experiments in low melting alloys, for example gallium-indium-tin (GalnSn) [1]. A suitable method for flow investigations in opaque media, where optical techniques are not applicable, is the pulse wave ultrasound Doppler velozimetry (PW-UDV) [2, 3]. But commercial off-the-shelf devices often employ only a limited number of transducers and are hardly suitable for mapping complex and unsteady flow phenomena.
We present an ultrasound array Doppler velocimeter (UADV) system for measuring flow fields in opaque media, which uses four ultrasound (US) line arrays. It provides high temporal and spatial resolution suitable to investigate complex transient flows. The capabilities of the UADV system are demonstrated by two measurements in magnetically stirred GalnSn.

\section{Sensors}

The measurement system utilizes US line arrays in order to obtain a one-component velocity measurement in a plane. An array consists of 25 single element piezo transducers $(2.5 \times 5 \mathrm{~mm})$ with a total sensitive length of $67 \mathrm{~mm}$ (fig. 1). The active elements are driven pairwise to form a square transducer of approx. $5 \times 5 \mathrm{~mm}$. Those dimensions are the result of a trade-off between element size and beam divergence and determine the lateral resolution to approx. $3 \mathrm{~mm}$ in GalnSn. This corresponds with the pitch of $2.7 \mathrm{~mm}$ that transducer pairs of the line array can be traversed electronically. 
The piezo-transducers are excited by a burst signal of eight sine periods at $f=8 \mathrm{MHz}$, which results in an axial resolution of about $1.4 \mathrm{~mm}$ in GalnSn [4].

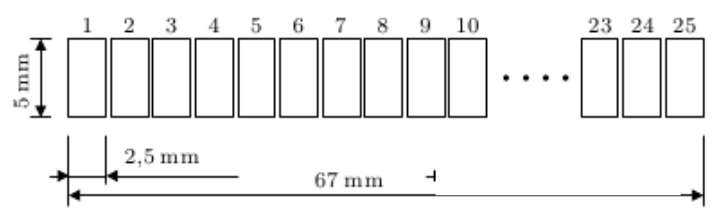

Figure 1: Linear ultrasound array with 25 transducer elements [4]

\section{Time division multiplex}

To obtain a high temporal resolution it is necessary to parallelize the measurement process along the line array. Therefore a time division multiplex (TDM) scheme is used to drive several transducer pairs simultaneously in order to measure multiple lines at once. It has been determined that a spatial distance of four inactive transducers and a temporal separation of one time step is sufficient to neglect crosstalk in GalnSn [4]. The outcome is an excitation pattern (fig. 2) that allows to scan a plane in $N_{s}=6$ time steps with an overall frame rate up to $30 \mathrm{~Hz}$ [6].

In order to obtain two or more velocity components, multiple sensor arrays measure in overlapping planes and are driven mutually exclusive. This is achieved by interleaving the excitation patterns of the sensor arrays. To measure a two-component (2c) velocity field along two planes (2.5d) for instance, two arrays are arranged orthogonally per plane and are electronically traversed in an alternating manner. Therefore twice the time is needed to completely capture a plane in a two-component configuration.

transducer element

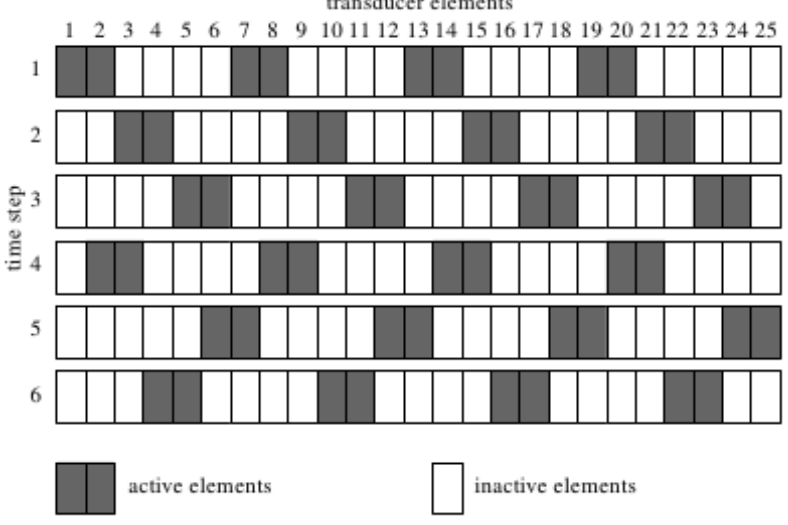

Figure 2: Time division multiplex scheme for a single line-array [5]

\section{Electrical Design}

The UADV employs a modular design that consist of an arbitrary function generator (AFG), a power amplifier, an electronic switching matrix and an analog-digital converter (ADC) card for each sensor array, see fig. 3 and fig. 4. Each AFG generates a burst signal, which is amplified and routed to four transducer pairs in one line array. The received echoes are separated from the burst signals, amplified with variable gain and converted into digital signals. The echo amplification is determined by a voltage ramp that is chosen to compensate for increasing attenuation with increasing time of flight of US-pulses in fluids (time gain compensation, TGC).

\section{Signal Processing}

The digitized echo signals are processed offline after acquisition by four ADC-cards to obtain the flow velocity profiles. The time-domain signal is processed through a finite impulse response (FIR) bandpass filter with $8 \mathrm{MHz}$ center frequency and $0.3 \mathrm{MHz}$ bandwidth. Subsequently the real-valued signal is complemented by a $90^{\circ}$ phase shifted imaginary part to form an analytic signal via the Hilbert transform. By sampling at specific time instances relative to the ultrasound burst emission a complex Doppler signal is derived. The mean frequency and directional information are subsequently estimated via an autocorrelation algorithm [7, 8]. The velocity of the fluid in the corresponding depth relative to the transducer is directly proportional to its Doppler frequency.

To obtain a spatial flow profile, the information of all line-arrays is combined according to their respective geometric positions and directions. A post-processing algorithm allows all orthogonal sensor configurations and provides the flexibility to measure for example multi-plane one (1c) and two-component $(2 \mathrm{c})$ velocity profiles as well as three velocity components (3c) along a line of three intersecting planes.

\section{Measurements in Liquid Metals}

To demonstrate the capabilities of the UADV system, measurements are performed in magnetically stirred GalnSn. The metal melt is contained in a cubic vessel of $67 \times 67 \times 67 \mathrm{~mm}$ that has several mounting options for US line arrays. An ultrasound pulse repetition frequency of $538 \mathrm{~Hz}$ is used to obtain 30 profiles of 50 echoes each, which are temporally averaged subsequently.

In a dual-plane setup, four sensor arrays span two measurement planes to allow quasisimultaneous capturing of two velocity 
components (2c). Arrays spanning the same plane are driven mutually exclusive, the crosstalk between two planes can be neglected ( $\Delta z \geq 15 \mathrm{~mm}$ ). The resulting $2.5 \mathrm{~d}-2 \mathrm{c}$ flow field is depicted in fig. 5 .

Three velocity components (3c) can be measured by intersecting three orthogonal single-component planes. With four available sensor arrays, three-component measurements along two lines are possible. Fig. 6 shows the experimental setup and the obtained flow field.

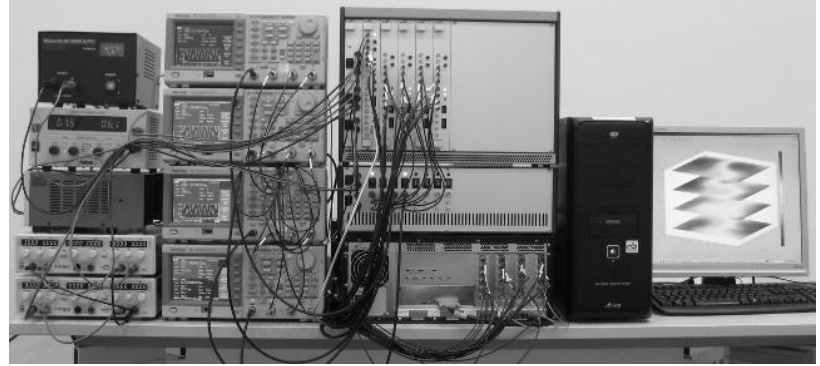

Figure 3: UADV system, from left to right: power supplies; arbitrary function generators (AFG); modular multiplex electronics; power amplifiers and signal acquisition; standard PC [6]

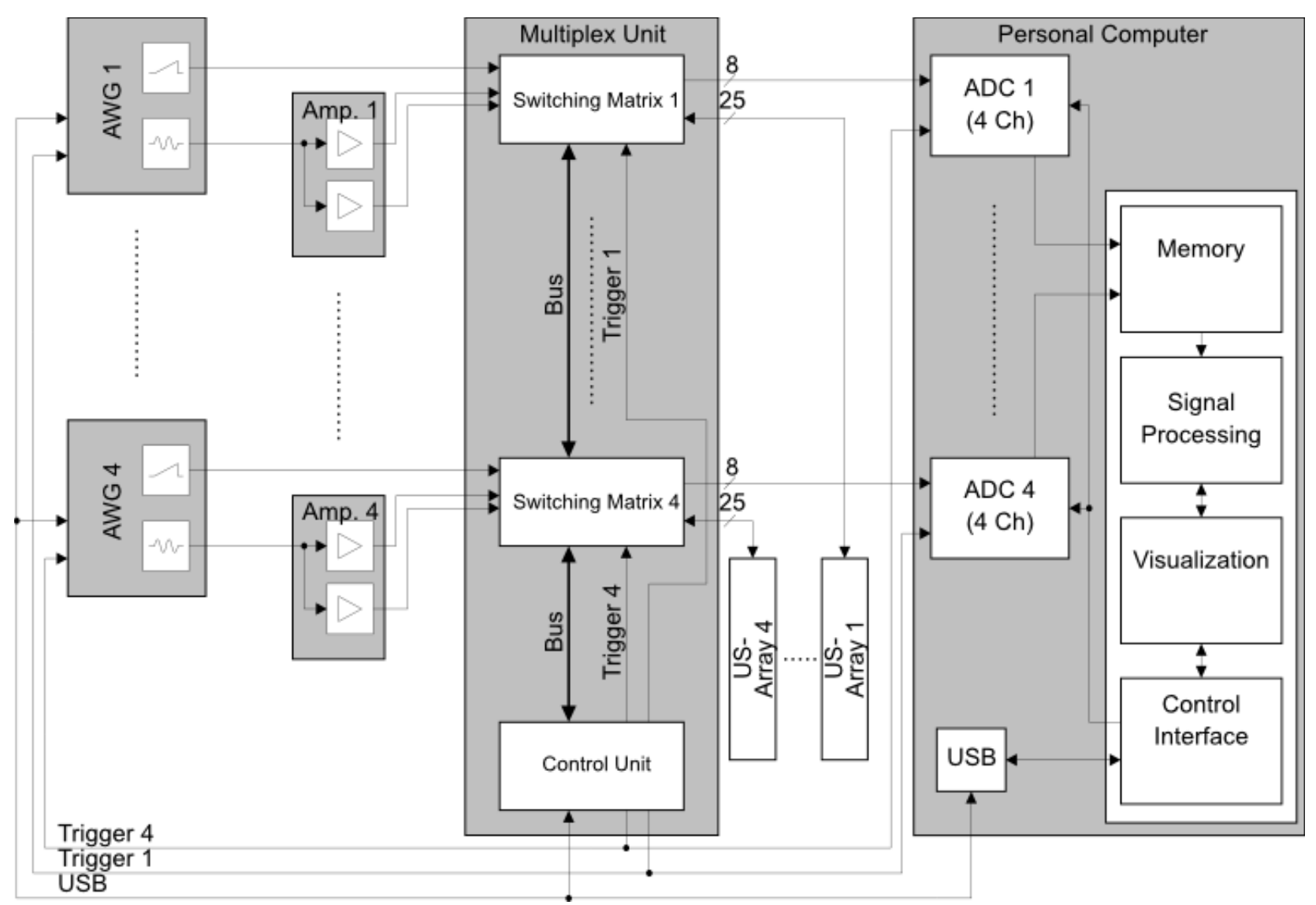

Figure 4: Block diagram of the UADV system [6] 

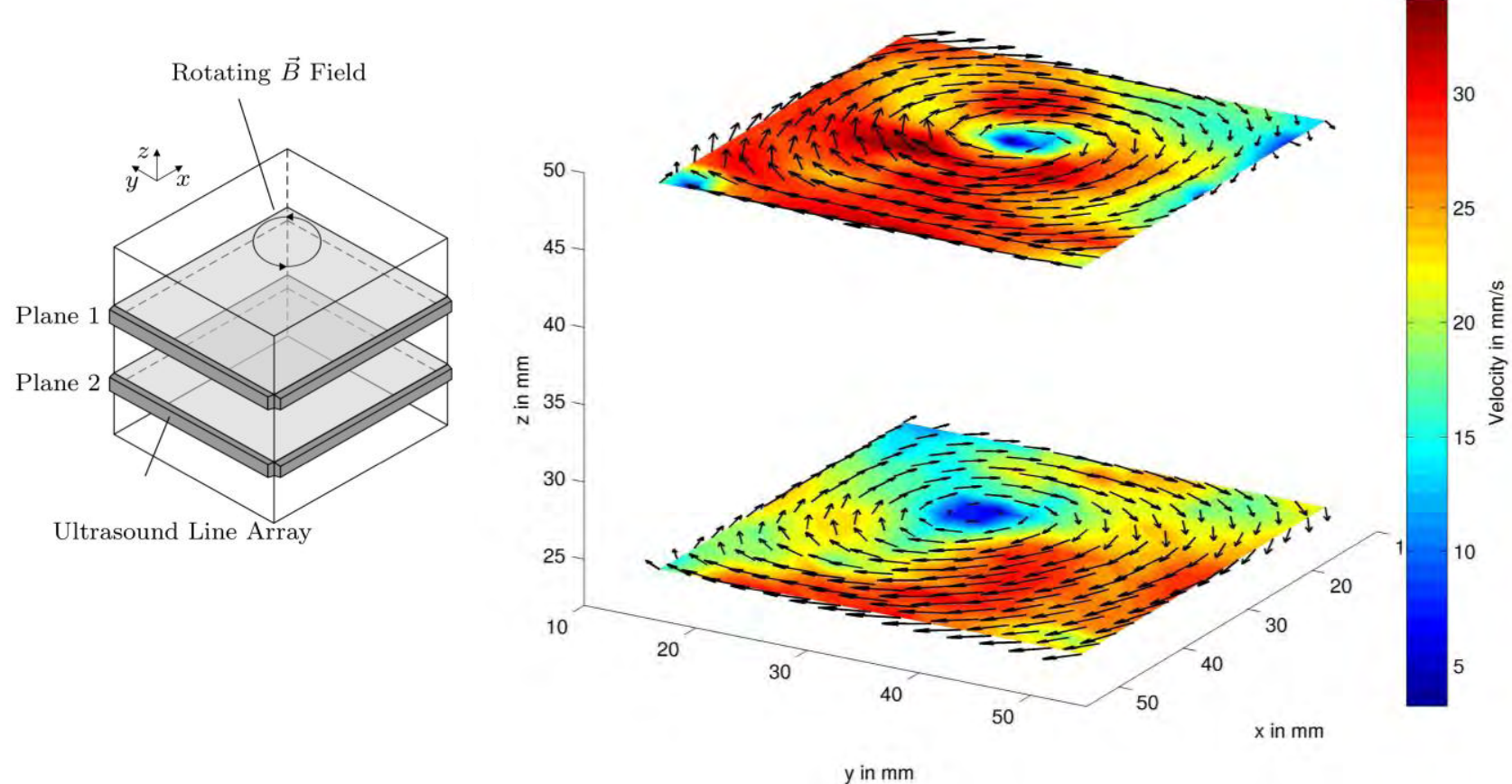

Figure 5: Dual-plane measurement $(2.5 d-2 c)$ : Measurement setup and results for magnetically stirred GalnSn in a cubic vessel [6]
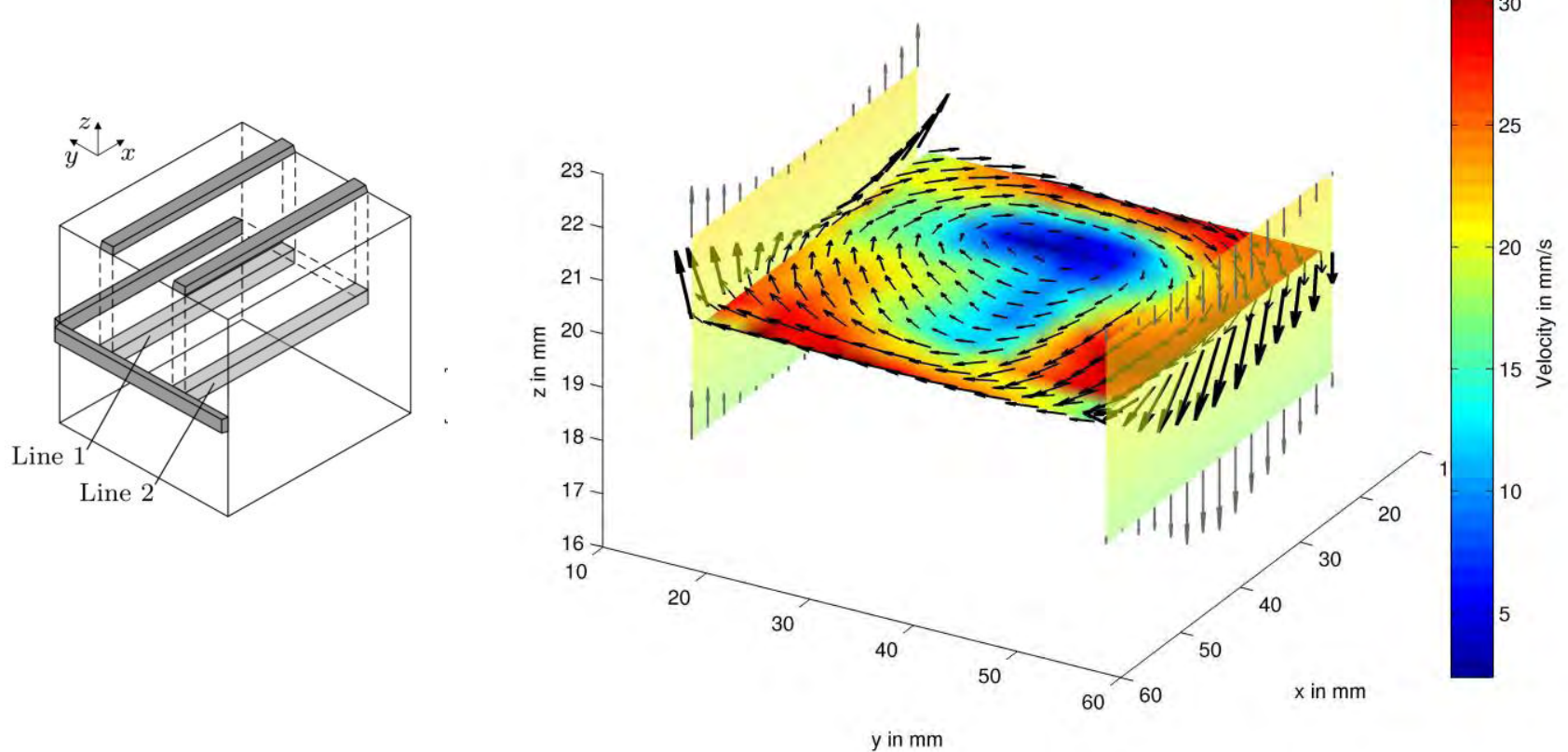

Figure 6: Dual-line measurement (1.5d - 3c): Measurement setup and results for magnetically stirred GalnSn in a cubic vessel; three-component vectors along two intersection lines are drawn bold [6] 


\section{Summary and Outlook}

The UADV system has been proven to be a capable tool for mapping complex flow patterns in opaque fluids. Compared to commercially available systems, it combines high spatial (up to $3 \mathrm{~mm}$ ) with high temporal resolution (up to $30 \mathrm{~Hz}$ ) due to optimized sensor design and fast time-division-multiplex. Its modular design allows for multi-plane and multi-component flow mapping. The system was demonstrated with a dual-plane $(2.5 \mathrm{~d}-2 \mathrm{c})$ and a three-component $(1.5 \mathrm{~d}-3 \mathrm{c})$ velocity measurement in a low melting liquid metal alloy.

The applications of the UADS system are manifold, especially in the field of MHD, where for instance measurements motivated by semiconductor crystal growth and continuous steel casting were performed [6].

Future development aims to extend the specifications of the UADV system: Improvements in signal processing have the potential to measure higher velocities and further increase the temporal resolution. Sensors able to withstand higher temperatures would enable experiments that include not only the interactions of the magnetic field and the fluid, but also thermal convection and phase boundaries. This is especially interesting for accurately modeling semiconductor crystal growth processes, e.g. in photovoltaics.

\section{Acknowledgement}

The authors would like to thank the Deutsche Forschungsgemeinschaft (DFG) for financial support from the grant "SFB 609 Electromagnetic Flow Control in Metallurgy, Crystal Growth and Electrochemistry."

\section{References}

[1] S. Eckert, G. Gerbeth, D. Räbiger, B. Willers, and C. Zhang, "Experimental modeling using low melting point metallic melts: Relevance for metallurgical engineering," Steel Res. Int., pp. 419-425, 2007.

[2] S. Eckert, A. Cramer, and G. Gerbeth, "Velocity measurement techniques for liquid metal flows," in Magnetohydrodynamics, vol. 80 of Fluid Mechanics and Its Applications, pp. 275-294, Springer Netherlands, 2007. 10.1007/978-14020-4833-3_17.

[3] Y. Takeda, "Development of an ultrasound velocity profile monitor," Nucl. Eng. Des., vol. 126, no. 2, pp. 277 - 284, 1991.

[4] S. Franke, L. Büttner, J. Czarske, D. Räbiger, and S. Eckert, "Ultrasound Doppler system for two-dimensional flow mapping in liquid metals," Flow Measurement and Instrumentation, vol. 21, pp. 402-409, 2010.
[5] S. Franke, H. Lieske, A. Fischer, L. Büttner, J. Czarske, D. Räbiger, and S. Eckert, "Twodimensional ultrasound doppler velocimeter for flow mapping of unsteady liquid metal flows," Ultrasonics, 2012.

[6] R. Nauber, L. Büttner, M. Burger, M. Neumann, J. Czarske, S. Franke, and S. Eckert, "Dual-plane ultrasound array doppler velocimeter for flow investigations in liquid metals," in 8th International Symposium on Ultrasonic Doppler Methods for Fluid Mechanics and Fluid Engineering, September 19-21, 2012, Dresden, Germany, pp. 7-10, 2012.

[7] A. Jensen, Estimation of blood velocities using ultrasound: a signal processing approach. Cambridge University Press, 1996.

[8] C. Kasai, K. Namekawa, A. Koyano, and R. Omoto, "Real-time two-dimensional blood flow imaging using an autocorrelation technique," Sonics and Ultrasonics, IEEE Transactions on, vol. 32, no. 3, pp. $458-464,1985$. 